\title{
Investigating structural subdivisions of the anterior cingulate cortex in schizophrenia, with implications for treatment resistance and glutamatergic levels
}

\author{
Ryo Ochi, BA; Eric Plitman, PhD; Raihaan Patel, BASc; Ryosuke Tarumi, MD, PhD; \\ Yusuke Iwata, MD, PhD; Sakiko Tsugawa, MD; Julia Kim, MSc; Shiori Honda, MA; \\ Yoshihiro Noda, MD, PhD; Hiroyuki Uchida, MD, PhD; Gabriel A. Devenyi, PhD; \\ Masaru Mimura, MD, PhD; Ariel Graff-Guerrero, MD, PhD*; \\ M. Mallar Chakravarty, PhD*; Shinichiro Nakajima, MD, PhD* $^{*}$
}

\begin{abstract}
Background: Abnormalities in the anterior cingulate cortex (ACC) are thought to play an important role in the pathophysiology of schizophrenia. Given regional variations in ACC structure, the present study aimed to examine ACC structural subdivisions and their relationships to treatment resistance and glutamatergic levels in schizophrenia. Methods: This study included 100 patients with schizophrenia and 52 healthy controls from 2 cohorts. We applied non-negative matrix factorization to identify accurate and stable spatial components of ACC structure. Between groups, we compared ACC structural indices in each spatial component based on treatment resistance or response and tested relationships with ACC glutamate + glutamine levels. Results: We detected reductions in cortical thickness and increases in mean diffusivity in the spatial components on the surface of the cingulate sulcus, especially in patients with treatment-resistant and clozapine-resistant schizophrenia. Notably, mean diffusivity in these components was higher in patients who did not respond to clozapine compared to those who did. Furthermore, these ACC structural alterations were related to elevated ACC glutamate + glutamine levels but not related to symptomatology or antipsychotic dose. Limitations: Sample sizes, cross-sectional findings and mixed antipsychotic status were limitations of this study. Conclusion: This study identified reproducible abnormalities in ACC structures in patients with treatment-resistant and clozapine-resistant schizophrenia. Given that these spatial components play a role in inhibitory control, the present study strengthens the notion that glutamate-related disinhibition is a common biological feature of treatment resistance in schizophrenia.
\end{abstract}

\section{Introduction}

Current antipsychotics fail to alleviate clinical symptoms in approximately one-third of patients with schizophrenia. ${ }^{1}$ This subtype is termed treatment-resistant schizophrenia, which is formally defined as having an inadequate response to 2 or more trials of first-line non-clozapine antipsychotics, despite sufficient dose and duration. ${ }^{2}$ Treatment-resistant schizophrenia represents a significant burden at the individual, familial and societal levels, and it is related to higher medical costs and a lower quality of life relative to the remission state. ${ }^{3}$ Given that most antipsychotic medications are antagonists or partial agonists of dopamine D2 receptors, ${ }^{4}$ neural components beyond dopamine may be fundamental neurobiological markers that contribute to explaining the pathophysiology of treatment-resistant schizophrenia.

Several studies have reported neurobiological abnormalities in the anterior cingulate cortex (ACC) of people in stages of illness that include the high-risk state, first-episode psychosis, chronic schizophrenia and treatment-resistant schizophrenia..$^{5-8}$ These studies suggested that abnormalities in this region may be fundamental to the pathophysiology of the schizophrenia spectrum. Thus, it is essential to increase our understanding of this critical region to improve treatment strategies.

The ACC has been implicated in various functions, such as decision-making, cognitive control and emotional processing. ${ }^{9,10}$ These diverse functions reflect regional variations in

Correspondence to: S. Nakajima, Department of Neuropsychiatry, Keio University School of Medicine, 35 Shinanomachi, Shinjuku-ku, Tokyo, Japan, 160-8582; shinichiro_nakajima@ hotmail.com

${ }^{*}$ A.G-G., M.M.C. and S.N. shared senior authorship.

Submitted Jul. 12, 2021; Revised Sep. 24, 2021; Accepted Oct. 25, 2021

Cite as: J Psychiatry Neurosci 2022 January 13;47(1). doi: 10.1503/jpn.210113 
ACC structure. ${ }^{9,11,12}$ The ACC can be divided into dorsal and rostral-ventral areas, which play key roles in cognitive and emotional processing, respectively. ${ }^{9,11,13}$ However, a comprehensive understanding of the different dimensions of ACC structure and its relationship to the pathophysiology of schizophrenia is still lacking. Notably, several studies using proton magnetic resonance spectroscopy ( $\left.{ }^{1} \mathrm{H}-\mathrm{MRS}\right)$ have reported elevated glutamatergic neurometabolite levels in the ACC in patients with treatment-resistant schizophrenia compared to patients with an adequate response to antipsychotics $^{14,15}$ or to healthy controls. ${ }^{5,6,16}$ The hyperglutamatergic state is hypothesized to induce neuronal overstimulation and increase intracellular calcium levels, resulting in a downstream cascade of events and ultimately leading to neuronal cell death or damage. ${ }^{17-19}$ Thus, glutamate-mediated excitotoxicity may contribute to structural alterations in patients with treatment-resistant schizophrenia.

In this study, we sought to investigate the complex structural architecture of the ACC in treatment-resistant or treatmentresponsive patients with schizophrenia and healthy controls. We adopted the approach recently described by Patel and colleagues ${ }^{20}$ in which they used non-negative matrix factorization (NMF) to investigate microstructural patterns in the hippocampus - a complex neuroanatomical region. The advantage of NMF is non-negative constraint, which allows for a purely additive reconstruction and the ability to describe data as a sum of their parts. Components derived from NMF have improved specificity and reproducibility compared to commonly used methods such as principal component analysis and independent component analysis. ${ }^{21,22}$ To capture the structural characteristics of the ACC comprehensively, we used vertex-wise indices derived from multimodal MRI as inputs for NMF. Notably, in addition to standard structural indices such as cortical thickness and surface area, we included microstructural indices derived from diffusion MRI (fractional anisotropy and mean diffusivity) to further capture tissue microstructures. ${ }^{23}$ Patel and colleagues ${ }^{20}$ noted increased reproducibility of NMF when using data from multimodal MRI rather than data from a single modality. Furthermore, we aimed to examine the relationship between glutamatergic neurometabolite levels and neuroanatomical characteristics of the ACC. We hypothesized that any neuroanatomical characteristics that were altered in the treatment-resistant schizophrenia group compared to the other groups would be related to glutamatergic levels. We also explored whether parcellated ACC structure might be related to symptomatology and antipsychotic dose in patients with schizophrenia.

\section{Methods}

\section{Tokyo cohort}

The original study for the Tokyo cohort was approved by the ethics committees at Komagino Hospital and Keio University School of Medicine. ${ }^{6}$ All participants in this cohort were recruited between 2017 and 2018 at Komagino Hospital following the completion of an informed consent procedure. We included 73 participants based on the completion of
$T_{1}$-weighted and diffusion MRI scans: 23 patients who did not respond to non-clozapine first-line antipsychotics (patients with treatment-resistant schizophrenia), 24 patients who did respond to first-line antipsychotics (responders to first-line antipsychotics) and 26 healthy controls. Inclusion and exclusion criteria for each group are described elsewhere, ${ }^{6,24}$ and in Appendix 1, available at www.jpn.ca/lookup/ doi/10.1503/jpn.210113/tab-related-content. Briefly, we defined antipsychotic treatment response or resistance using the modified Treatment Response and Resistance in Psychosis Working Group Consensus criteria. ${ }^{2}$

All participants were scanned in a 3 T GE Signa HDxt scanner equipped with an 8-channel head coil, and $T_{1}$-weighted images were acquired (BRAVO; repetition time $6.4 \mathrm{~ms}$; echo time $2.8 \mathrm{~ms}$; inversion time $650 \mathrm{~ms}$; flip angle $8^{\circ}$; field of view $230 \mathrm{~mm}$; matrix $256 \times 256$; slice thickness $0.9 \mathrm{~mm}$ ). We collected ${ }^{1} \mathrm{H}-\mathrm{MRS}$ using point-resolved spectroscopy (repetition time 2000 ms; echo time 35 ms; spectral width $5000 \mathrm{~Hz} ; 4096$ data points; 128 water-suppressed and 16 water-unsuppressed averages; 8 numbers of excitation). Diffusion MRI data consisted of 30 diffusion-weighted volumes with different noncollinear diffusion directions, all with a $b$ value of $1000 \mathrm{~s} / \mathrm{mm}^{2}$, and 5 diffusion-unweighted volumes with a $b$ value of $0 \mathrm{~s} / \mathrm{mm}^{2}$ (repetition time $16000 \mathrm{~ms}$; echo time $71 \mathrm{~ms}$; flip angle $90^{\circ}$; matrix $128 \times 128$; slice thickness $2.5 \mathrm{~mm}$ ).

\section{Toronto cohort}

The original study from Toronto was approved by the research ethics board at the Centre for Addiction and Mental Health. ${ }^{5}$ All participants in this cohort were recruited between 2014 and 2018 at the Centre for Addiction and Mental Health following the completion of an informed consent procedure. We included 79 participants based on the completion of $T_{1}$-weighted and diffusion MRI scans: 21 patients who did not respond to first-line antipsychotics and clozapine (patients with ultra-treatment-resistant schizophrenia), 15 patients who responded to clozapine (responders to clozapine), 17 responders to first-line antipsychotics and 26 healthy controls. Definitions of treatment response or resistance are described elsewhere ${ }^{5,25,26}$ and in Appendix 1. In brief, as with the Tokyo cohort, we defined antipsychotic treatment response or resistance using the modified Treatment Response and Resistance in Psychosis Working Group Consensus criteria. ${ }^{2}$

We obtained $T_{1}$-weighted images of all participants at the Centre for Addiction and Mental Health using a 3 T GE Discovery MR750 scanner equipped with an 8-channel head coil (repetition time $6.74 \mathrm{~ms}$; echo time $3.00 \mathrm{~ms}$; inversion time $650 \mathrm{~ms}$; flip angle $8^{\circ}$; field of view $230 \mathrm{~mm}$; matrix $256 \times 256$; slice thickness $0.9 \mathrm{~mm}$ ). We obtained ${ }^{1} \mathrm{H}-\mathrm{MRS}$ data using point-resolved spectroscopy (repetition time $2000 \mathrm{~ms}$; echo time $35 \mathrm{~ms}$ ). Diffusion MRI data consisted of 30 diffusionweighted volumes with different noncollinear diffusion directions, all with a $b$ value of $1000 \mathrm{~s} / \mathrm{mm}^{2}$, and 3 diffusionunweighted volumes with a $b$ value of $0 \mathrm{~s} / \mathrm{mm}^{2}$ (repetition time $8800 \mathrm{~ms}$; echo time $82.4 \mathrm{~ms}$; flip angle $90^{\circ}$; matrix $128 \times$ 128; slice thickness $2.0 \mathrm{~mm}$ ). 


\section{Clinical assessment}

We assessed symptom severity using the Positive and Negative Syndrome Scale ${ }^{27}$ and the Clinical Global Impression Severity Scale. ${ }^{28}$ We assessed cognitive function using the Repeatable Battery for the Assessment of Neuropsychological Status. ${ }^{29}$

\section{Data preprocessing}

All participants completed $3 \mathrm{~T}$ MRI scans. We visually inspected all images for quality. We preprocessed $T_{1}$-weighted images using the minc-bpipe-library pipeline (https:/ /github. com/CobraLab/minc-bpipe-library), including N4 correction ${ }^{30}$ and neck cropping. We performed diffusion MRI data preprocessing (including denoising ${ }^{31}$ and motion and eddy current distortion correction ${ }^{32}$ ) with Mrtrix3 software. ${ }^{33}$ After tensor fitting, we estimated fractional anisotropy and mean diffusivity maps at each voxel using Mrtrix3's tensor2metric function. ${ }^{34}$

In both cohorts, ${ }^{1} \mathrm{H}$-MRS voxels were positioned on an oblique sagittal image acquired parallel to the anterior commissure-posterior commissure line and an oblique sagittal image acquired parallel to the head midline (voxel size $9.0 \mathrm{~mL}$ ). The tip of the voxel was placed on top of the most anterior part of the genu, parallel to the cingulate cortex (for details, see Appendix 1, Figure S1). Details of the ${ }^{1} \mathrm{H}-\mathrm{MRS}$ analyses are described in our previous studies. ${ }^{5,6}$ Briefly, we estimated metabolite levels using LCModel..$^{35}$ Then, we corrected for partial volume effects using tissue volume fractions determined from segmented $T_{1}$-weighted images. Spectra with signal-to-noise ratios of 10 or less, full-width at half maximum of $10 \mathrm{~Hz}$ or more or percentage standard deviation values of $20 \%$ or more were deemed to be of poor quality and excluded from subsequent analyses. Among the participants whose data were included, we found no group differences in these measures. In the present study, the neurometabolite of interest was glutamate + glutamine (Glx), because our parameters were not optimized for separating them. ${ }^{36,37}$

\section{Extracting structural indices in the ACC}

Detailed procedures to obtain neuroanatomical indices are described in Appendix 1. Briefly, we performed cortical surface extraction using the CIVET processing pipeline (version 2.1.0; Montreal Neurological Institute), resulting in 40962 vertices per hemisphere. We delineated a binary ACC mask using the combination of caudal and rostral ACC defined by the Desikan Killiany-Tourville atlas, ${ }^{38}$ resulting in 1694 vertices (847 vertices per hemisphere). We extracted cortical thickness, surface area, mean diffusivity and fractional anisotropy values for each vertex in the mask and used them as inputs for the NMF analysis.

\section{Non-negative matrix factorization}

In this study, we used an orthogonal variant of NMF, orthogonal projective NMF (OPNMF). ${ }^{21,39}$ Details are described in Appendix 1. Briefly, OPNMF decomposes a given input matrix of dimensions $m \times n$ into a component matrix $(m \times k)$ and a weight matrix $(k \times n)$, in which $k$ is the number of components.
These matrices are generated such that their multiplication reconstructs the input matrix as well as possible by minimizing the reconstruction error between the original and reconstructed inputs. In the present study, we performed OPNMF on an input matrix that contained vertex-wise cortical thickness, surface area, mean diffusivity and fractional anisotropy values for the bilateral ACC (separately for each cohort).

To determine the optimal number of components, we assessed both the accuracy and stability of OPNMF performance, following procedures in the literature. ${ }^{20} \mathrm{We}$ assessed the former using the gradient of reconstruction error, and the latter using the similarity of output spatial components across varying splits of data. After splitting each cohort into 2 based on age and disease categories, we performed OPNMF on each subgroup independently to obtain the component matrix of each. We calculated the stability index as the average correlation coefficient across each row of the cosine similarity matrix of output spatial components between the 2 splits; 1 represented perfect stability, and -1 represented instability. We repeated the above procedure for 10 random splits of data, for 2 to 10 components. To evaluate the reproducibility of OPNMF performance, we estimated the overlap between the OPNMF outputs of the 2 cohorts using an adjusted Rand index. An adjusted Rand index of 1 indicates perfect consistency of clustering performance; 0 indicates inconsistency. Because we found that age and sex were related to structural indices parcellated by a 4-component solution (for details, see Appendix 1), we controlled the statistical analyses in this study by age and sex.

\section{Statistical analysis}

We compared demographic and clinical characteristics between groups using analyses of variance for continuous variables or $\chi^{2}$ tests for categorical variables.

First, we tested group differences of structural indices in each parcellated region using analysis of covariance, controlling for age and sex. We then conducted post hoc comparisons for indices in which analysis of covariance reached the level of significance in both cohorts. We restricted subsequent analyses by using ACC structural indices that showed significant group differences in both cohorts. Second, to test glutamatergic effects on structural alterations in treatmentresistant schizophrenia, we tested the relationship between ACC structural indices and Glx, as well as their interactions with the groups, using analysis of covariance and controlling for age and sex. If we identified significant relationships, we performed follow-up partial correlation analyses, controlling for age and sex. Third, using linear models, we explored the relationships between ACC structural indices and clinical measures, including subscale scores from the Positive and Negative Syndrome Scale (positive, negative and general psychopathology), total score from the Repeatable Battery for the Assessment of Neuropsychological Status, age at onset, illness duration, chlorpromazine equivalent daily dose ${ }^{40}$ and clozapine daily dose in patients with schizophrenia. Because most of the responders to first-line antipsychotics and responders to clozapine were in remission, 
we tested relationships with Positive and Negative Syndrome Scale subscales only in patients with treatmentresistant or ultra-treatment-resistant schizophrenia. As well, we restricted testing of relationships with clozapine daily dose to only responders to clozapine and patients with ultratreatment-resistant schizophrenia in the Toronto cohort. All analyses were corrected for multiple comparisons using false discovery rate (FDR) correction. Only FDR-corrected $p$ values are reported in the present study.

\section{Results}

\section{Participant demographics}

In total, we included 100 patients with schizophrenia and 52 healthy controls from the 2 cohorts. Participants' demographics and clinical characteristics are shown in Appendix 1, Tables S1 and S2. The total score on the Repeatable Battery for the Assessment of Neuropsychological Status was different among patients with treatment-resistant schizophrenia, responders to first-line antipsychotics and healthy controls in the Tokyo cohort, as well as among patients with ultra-treatment-resistant schizophrenia, responders to clozapine, responders to first-line antipsychotics and healthy controls in the Toronto cohort. Among patients in the Tokyo cohort, total and all subscale scores on the Positive and Negative Syndrome Scale, score on the Clinical Global Impression Severity Scale and chlorpromazine equivalent daily dose were higher in those with treatment-resistant schizophrenia than in responders to first-line antipsychotics. In the Toronto cohort, these variables were higher in those with ultra-treatment-resistant schizophrenia than in responders to clozapine and responders to first-line antipsychotics. As well, clozapine daily doses were higher in those with ultra-treatment-resistant schizophrenia than in responders to clozapine.

\section{Non-negative matrix factorization}

The results of the stability analysis are shown in Appendix 1, Figure S2. In both cohorts, stability indices at $k=4$ were above 0.8 . Moving from $k=4$ to $k=5$, we observed dramatic drop-offs in the stability index. For this reason, the 4-component solution was deemed a suitable choice for observing complex yet stable spatial patterns. In terms of accuracy, the gain in accuracy when moving from $k=4$ to $k=5$ was much less than when moving from $k=3$ to $k=4$. For $k$ greater than 5 , we observed a smaller change in the gradient from one component to the next. These observations suggested that at $k=4$, the most prominent patterns were captured and that any added complexity had a diminishing return on reconstruction accuracy. Accordingly, we used the 4-component solution for the subsequent analysis. Figure 1 displays the 4-component solution for the ACC. The performance of OPNMF-derived spatial components was consistent between the Tokyo and Toronto cohorts (adjusted Rand index $=0.80$ ).

\section{A Structure-based parcellation using the Tokyo cohort}
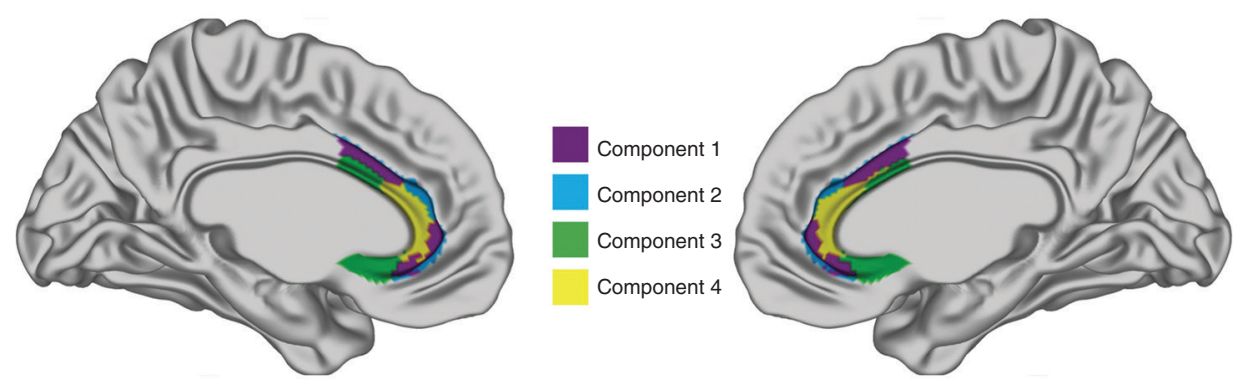

B Structure-based parcellation using the Toronto cohort
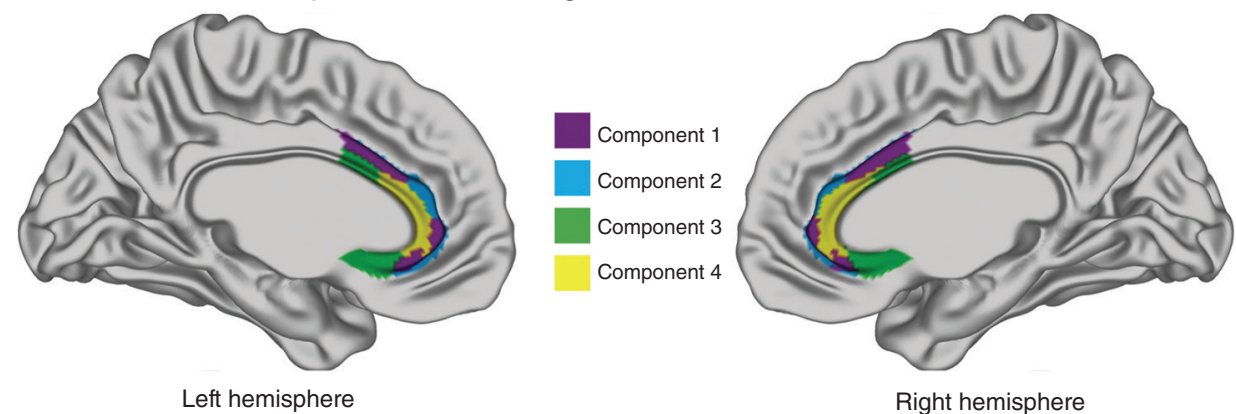

Figure 1: Four-component solution for the ACC structure of $(A)$ the Tokyo cohort and $(B)$ the Toronto cohort. The panels display sagittal views of each $\mathrm{ACC}$ component for the 2 cohorts. $\mathrm{ACC}=$ anterior cingulate cortex. 


\section{Group comparisons of ACC structural indices}

In the Tokyo cohort, the cortical thickness and mean diffusivity of all ACC spatial components differed significantly among groups (Appendix 1, Table S3). In the Toronto cohort, the cortical thickness and mean diffusivity of components 1 and 2 were significantly different among groups (Appendix 1, Table S4). Taken together, the cortical thickness and mean diffusivity of components 1 and 2 were different among groups in both cohorts. Figure 2 displays comparisons of the cortical thickness and mean diffusivity for components 1 and 2 between groups.

Post hoc comparisons revealed that in the Tokyo cohort, the cortical thickness of components 1 and 2 was reduced in patients with treatment-resistant schizophrenia (component 1 : $t=4.18, p<0.001$; component $2: t=5.10, p<0.001$ ) and responders to first-line antipsychotics (component 1: $t=3.83$, $p<0.001$; component $2 ; t=4.29, p<0.001$ ) compared to healthy controls. As well, the mean diffusivity of component 1 was increased in responders to first-line antipsychotics $(t=$ $2.58, p=0.02$ ) and patients with treatment-resistant schizophrenia $(t=3.55, p=0.003)$ relative to healthy controls; the mean diffusivity of component 2 was increased only in patients with treatment-resistant schizophrenia $(t=3.05, p=0.011)$.

In the Toronto cohort, the cortical thickness of both components was reduced in patients with ultra-treatmentresistant schizophrenia (component $1: t=3.58, p=0.003$; component 2: $t=4.11, p<0.001)$ and in responders to clozapine (component 1: $t=3.57, p=0.003$; component 2: $t=4.36, p<$ $0.001)$ compared to healthy controls. The mean diffusivity of both components was increased in those with ultratreatment-resistant schizophrenia compared to responders to clozapine (component 1: $t=2.64, p=0.039$; component 2: $t=$ 2.93, $p=0.018$ ), and to healthy controls (component $1: t=$ 2.75, $p=0.039$; component 2: $t=2.97, p=0.018)$.

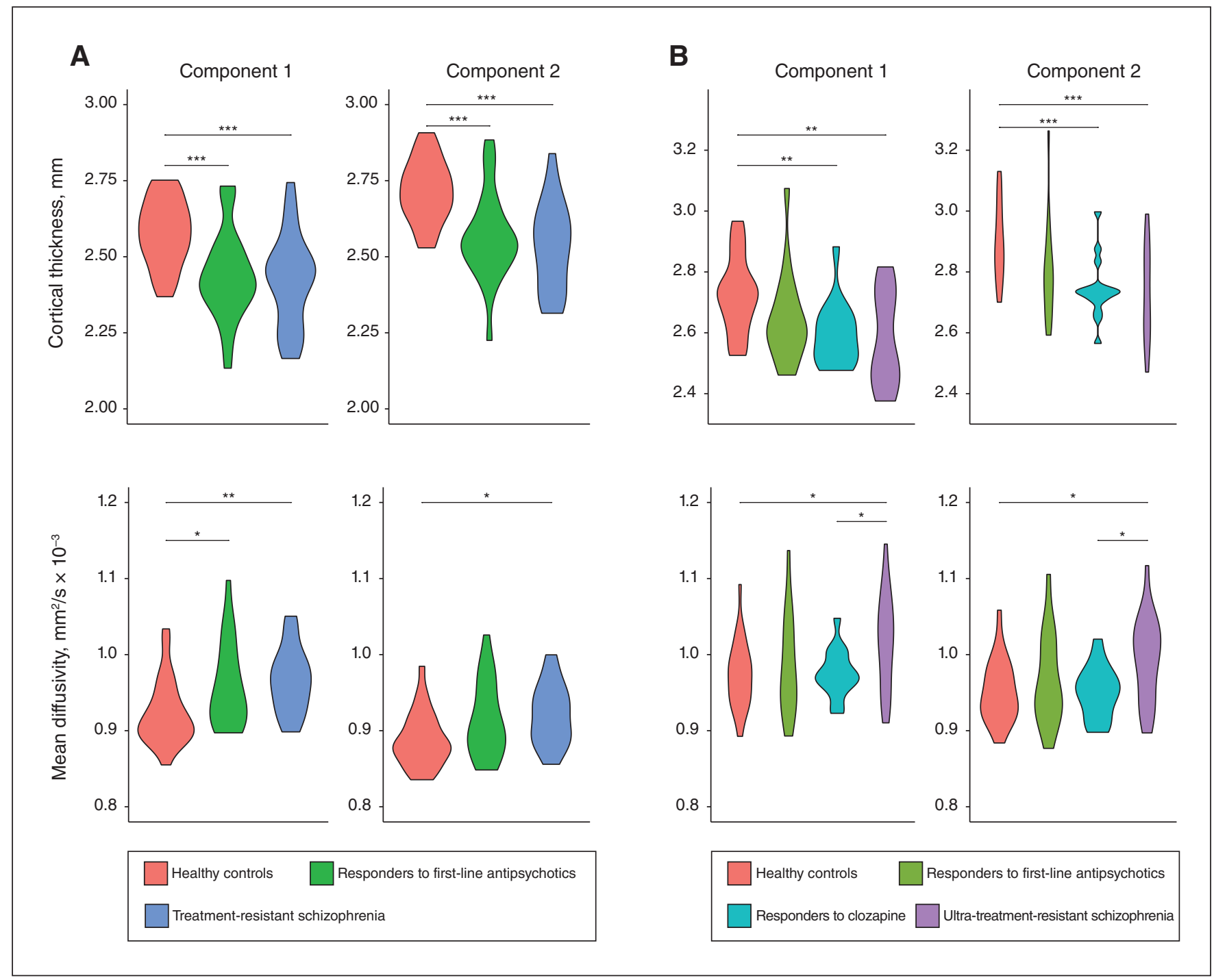

Figure 2: Significant differences of cortical thickness and mean diffusivity in spatial components 1 and 2 of the ACC in (A) the Tokyo cohort and (B) the Toronto cohort. ${ }^{*} p<0.05 ;{ }^{* *} p<0.01 ;{ }^{* * *} p<0.001$. ACC $=$ anterior cingulate cortex. 


\section{Relationships with Glx levels}

After performing quality assurance of the ${ }^{1} \mathrm{H}-\mathrm{MRS}$ data, we excluded 8 participants from the Tokyo cohort (3 with treatment-resistant schizophrenia, 4 responders to firstline antipsychotics and 1 healthy control) from the analysis. No participants were excluded from the Toronto cohort. Although the present study included only some participants who completed multimodal MRI, we confirmed elevated Glx levels in patients with treatment-resistant schizophrenia relative to healthy controls $(p=0.005)$ and in patients with ultra-treatment-resistant schizophrenia relative to healthy controls $(p=0.038)$, in line with previous studies. ${ }^{5,6}$ Analyses revealed that increased cortical thickness and reduced mean diffusivity in components 1 and 2 were related to ACC Glx levels (Figure 3). In the
Tokyo cohort, we found that the cortical thickness of components 1 and 2 and the mean diffusivity of component 1 were significantly related to ACC Glx levels (cortical thickness of component 1: $r=-0.396, p=0.004$; cortical thickness of component 2: $r=-0.474, p<0.001$; mean diffusivity of component 1: $r=0.217, p=0.049$ ). The mean diffusivity of component 2 did not reach significance $(r=0.202, p=$ 0.054). We did not find any group interactions for these relationships. As well, in the Toronto cohort, all indices that were significantly different among the groups were related to ACC Glx levels (cortical thickness of component $1: r=-0.212, p=0.007$; cortical thickness of component 2 : $r=-0.281, p=0.002$; mean diffusivity of component $1: r=$ $0.233, p=0.002$; mean diffusivity of component $2: r=0.244$, $p=0.002)$. We did not find any group interactions for these relationships.

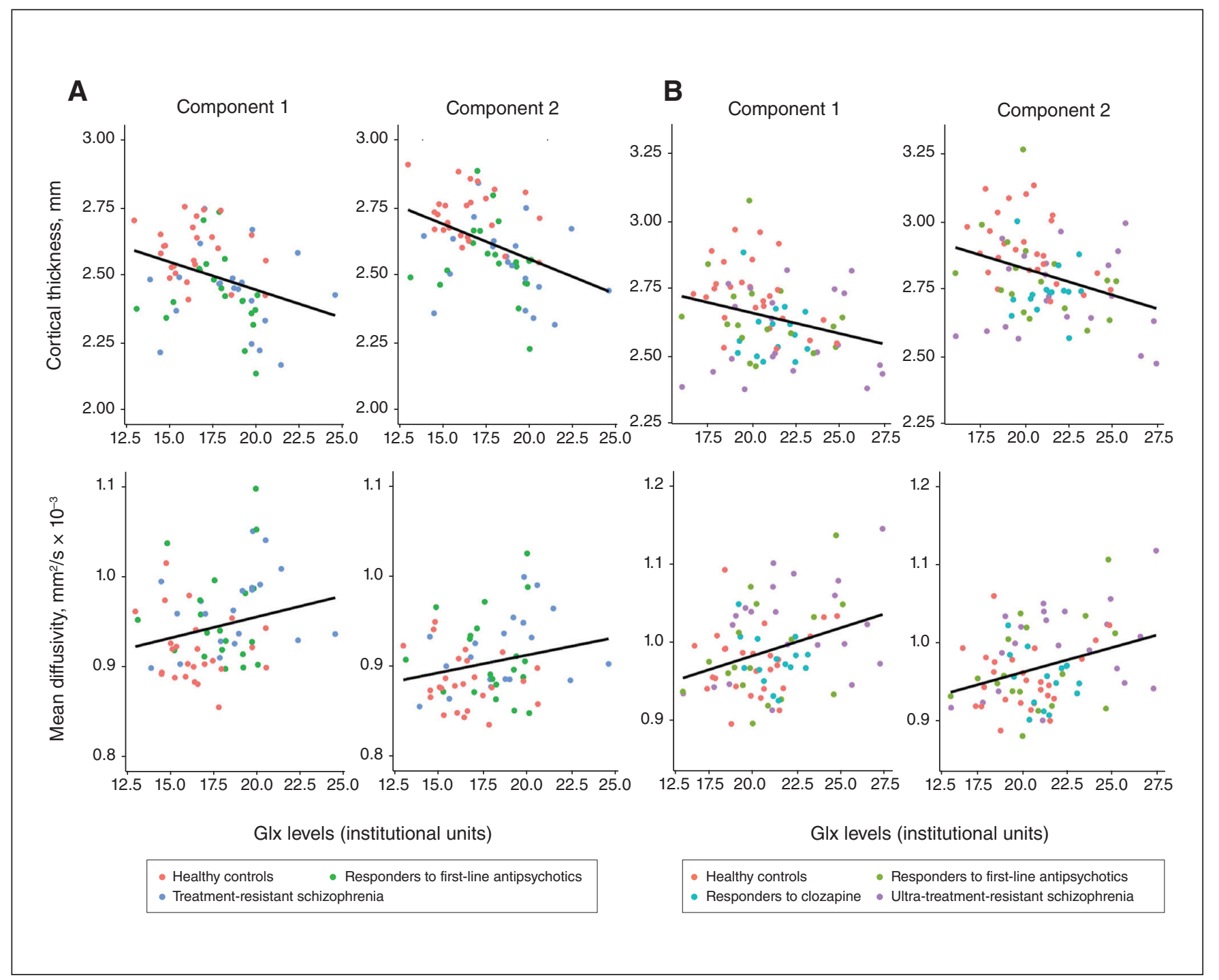

Figure 3: Relationships between cortical thickness and mean diffusivity in spatial components 1 and 2 of the ACC and Glx levels in (A) the Tokyo cohort and (B) the Toronto cohort. Black lines indicate fitted linear models between structural indices and Glx levels. ACC = anterior cingulate cortex; Glx, glutamate + glutamine. 


\section{Relationships with symptomatology and antipsychotic dose}

Our exploratory analyses found no relationships between the cortical thickness and mean diffusivity of components 1 and 2 and clinical variables, including symptomatology, age at onset, illness duration and antipsychotic dose. As well, consistent with previous studies, ${ }^{5,6}$ ACC Glx levels were not related to these clinical measures.

\section{Discussion}

In the present study, we sought to investigate patterns of ACC structure, as assessed by $T_{1}$-weighted and diffusion MRI, in treatment-resistant or treatment-responsive patients with schizophrenia and healthy controls using OPNMF. Based on a balance between stability and accuracy, we found 4 ACC spatial components that were reproducible between the 2 cohorts. In both cohorts, we found reduced cortical thickness and increased mean diffusivity of these spatial components, which were located on the surface of the cingulate sulcus, especially in patients with treatment-resistant schizophrenia and ultra-treatmentresistant schizophrenia relative to healthy controls. Furthermore, in the Toronto cohort, we found increased mean diffusivity in these components in patients with ultratreatment-resistant schizophrenia compared to responders to clozapine. As well, these alterations were related to elevated ACC Glx levels but not to symptomatology or antipsychotic dose.

Structural brain abnormalities in patients with schizophrenia are some of the most consistently reported findings in the schizophrenia neuroimaging literature. ${ }^{41-43}$ The ACC is the region commonly reported to show abnormal structure and function in patients with the disorder across the various stages of illness..$^{5-8}$ In line with these studies, we found reduced cortical thickness and increased mean diffusivity in ACC subdivisions on the surface of the cingulate sulcus in patients with treatment-resistant schizophrenia or ultra-treatment-resistant schizophrenia compared to healthy controls. These alterations were suggestive of increased synapse elimination and sparser axonal organizations in these components of the ACC in patients with treatment-resistant or ultra-treatment-resistant schizophrenia. ${ }^{23,44}$ Supporting this finding, previous studies have noted reduced synaptic density in the ACC in patients with schizophrenia. ${ }^{45,46}$ Analyses revealed that these alterations in patients with treatment-resistant schizophrenia or ultratreatment-resistant schizophrenia were only in components on the surface of the cingulate sulcus (components 1 and 2) but not in those on the surface of the cingulate gyrus (components 3 and 4). Evidence has suggested that areas on the surface of the cingulate gyrus play a role in interoception, and those on the surface of the cingulate sulcus play a role in the inhibition of action, pointing to functional differences in the region. ${ }^{12}$ Given the relationships we found with elevated Glx levels, our results suggest that glutamatergic dysfunction may lead to structural alterations in inhibitory control-related subdivisions of the ACC via the excitatory process. Limongi and colleagues demonstrated that glutamate levels in the dorsal ACC were negatively related to inhibitory influence on the excitatory population of the region in patients with first-episode psychosis. ${ }^{47}$ The present study strengthens the notion that disinhibition caused by glutamatergic dysfunction is a common biological feature of treatment resistance to antipsychotics, including clozapine.

In the Toronto cohort, we also found increased mean diffusivity in these components in patients with ultra-treatmentresistant schizophrenia compared to responders to clozapine. There are 2 possible mechanisms for this difference. First, the difference may exist before clozapine administration. A recent systematic review noted that higher prefrontal volumes before clozapine initiation are related to a better response to clozapine. ${ }^{48}$ Our results are in line with this notion, because mean diffusivity is thought to reflect microstructural disorganization and disruption of cellular membranes. ${ }^{49}$ Therefore, mean diffusivity in the area may be a surrogate marker of treatment response or resistance to clozapine. Second, successful clozapine treatment may reduce the mean diffusivity of components 1 and 2 in responders to clozapine. Although we did not find any relationship with clozapine dose, previous studies have shown that clozapine administration led to altered brain structure and inflammatory processes. ${ }^{50,51}$ Taken together with the differences in mean diffusivity we found between those with ultra-treatment-resistant schizophrenia and responders to clozapine, we cannot exclude the possibility that clozapine may alter brain structure only in responders to clozapine. Because no robust neuroimaging correlates of ultra-treatment-resistant schizophrenia have been found thus far, ${ }^{52}$ future longitudinal studies that assess patients with treatment-resistant schizophrenia before and after clozapine treatment are warranted.

We found no relationships between ACC structural indices and symptom severity, suggesting that symptom severity might not vary in association with ACC structures. On the other hand, the sample size of the 2 cohorts may not have been large enough to detect such relationships. Furthermore, clinical heterogeneity (e.g., different antipsychotics and medication adherence) might confound relationships; future studies are warranted.

\section{Limitations}

Our findings should be interpreted in light of several limitations. First, in both cohorts, the sample size of each group was relatively small. Future studies with a larger sample size may enable more precise parcellation. Second, each patient group consisted of patients taking different antipsychotics, which may have affected ACC structural organization differently. Third, diffusion MRI-derived indices (fractional anisotropy and mean diffusivity) are not specific to underlying biological changes. ${ }^{53}$ Future studies can take this into account by using advanced diffusion MRI-based modelling, such as neurite orientation dispersion and density imaging. ${ }^{54}$ Fourth, we did not assess detailed behavioural 
phenotypes of the participants, and this prevented us from inferring the functional properties of each component. Future studies are needed that link each component to broad behavioural dimensions to characterize functional properties. Finally, because of the nature of cross-sectional design and the greater clinical severity among those with treatmentresistant schizophrenia or ultra-treatment-resistant schizophrenia relative to other patient groups, we could not conclude whether our findings were a trait marker of treatment response or resistance in schizophrenia, or a state marker representing clinical severity in those with treatmentresistant or ultra-treatment-resistant schizophrenia.

\section{Conclusion}

Our main findings from the 2 cross-sectional cohorts were as follows: we found reduced cortical thickness and increased mean diffusivity in the spatial components on the surface of the cingulate sulcus in patients with treatment-resistant schizophrenia or ultra-treatment-resistant schizophrenia relative to healthy controls; we found increased mean diffusivity in these areas in patients with ultra-treatment-resistant schizophrenia compared to responders to clozapine; reduced cortical thickness and increased mean diffusivity in these components were related to elevated Glx levels in the ACC; and altered structural indices were not related to symptomatology or antipsychotic dose. Collectively, our results suggest that the hyperglutamatergic state may lead to disinhibition-related structural compromises seen in patients with treatment-resistant or ultra-treatment-resistant schizophrenia. Going forward, studies are warranted that use a prospective design in patients with treatment-resistant schizophrenia before and after clozapine administration. An improved understanding of the ACC structural abnormalities may aid in furthering our knowledge of the pathophysiology of schizophrenia.

Affiliations: From the Department of Neuropsychiatry, Keio University School of Medicine, Tokyo, Japan (Ochi, Tarumi, Tsugawa, Honda, Noda, Uchida, Mimura, Nakajima); the Cerebral Imaging Centre, Douglas Mental Health University Institute, Montreal, Que., Canada (Plitman, Patel, Devenyi, Chakravarty); the Department of Psychiatry, McGill University, Montreal, Que., Canada (Plitman, Devenyi, Chakravarty); the Department of Biological and Biomedical Engineering, McGill University, Montreal, Que., Canada (Patel, Chakravarty); the Multimodal Imaging Group, Research Imaging Centre, Centre for Addiction and Mental Health, Toronto, Ont., Canada (Iwata, Kim, Graff-Guerrero, Nakajima); and the Institute of Medical Science, University of Toronto, Toronto, Ont., Canada (Kim, Graff-Guerrero).

Funding: This work was supported by the Japan Society for the Promotion of Science (18H02755), Japan Research Foundation for Clinical Pharmacology, Japan Agency for Medical Research and Development (AMED), Naito Foundation, Takeda Science Foundation and Uehara Memorial Foundation (Noda, Mimura, Nakajima). The funding agency did not contribute to the study design; to the data collection, analyses and interpretation; to the writing of the manuscript; or to the decision to submit the manuscript for publication.

Competing interests: E. Plitman has received research support from an Ontario Graduate Scholarship (OGS), a Canadian Institutes of Health Research (CIHR) Canada Graduate Scholarship-Master's, a CIHR Vanier Canada Graduate Scholarship and the Healthy
Brains for Healthy Lives Postdoctoral Fellowship. Y. Iwata has received fellowship grants from the CIHR, research support from the Japan Society for the Promotion of Science, the Japanese Society of Clinical Neuropsychopharmacology, Inokashira Hospital Grants for psychiatry research, manuscript fees from Dainippon Sumitomo Pharma and speaker's fees from Eli Lilly, Meiji-Seika Pharma, Mochida Pharmaceutical and Yoshitomi Yakuhin within the past 3 years. Y. Noda has received a Grant-in-Aid for Young Scientists (KAKENHI); research grants from AMED; an investigator-initiated clinical study grant from Teijin Pharma Ltd.; research grants from the Japan Health Foundation, the Meiji Yasuda Mental Health Foundation, the Mitsui Life Social Welfare Foundation, the Takeda Science Foundation, the Senshin Medical Research Foundation, the Health Science Center Foundation, the Mochida Memorial Foundation for Medical and Pharmaceutical Research, the Taiju Life Social Welfare Foundation, and Daiichi Sankyo Scholarship Donation Program; and equipment-in-kind support for an investigator-initiated study from Magventure Inc, Inter Reha Co., Ltd., Rogue Resolutions Ltd. and Miyuki Giken Co., Ltd. H. Uchida has received grants from Eisai, Otsuka Pharmaceutical, Dainippon-Sumitomo Pharma, Mochida Pharmaceutical, Meiji-Seika Pharmaceutical and Novartis; speaker's honoraria from Otsuka Pharmaceutical, Eli Lilly, Shionogi, Pfizer, Yoshitomi Yakuhin, Dainippon-Sumitomo Pharma, Meiji-Seika Pharma, MSD and Janssen Pharmaceutical; and advisory panel payments from Dainippon-Sumitomo Pharma within the past 3 years. M. Mimura has received research support from the Japan Society for the Promotion of Science and grants or speaker's honoraria from Daiichi Sankyo, Dainippon-Sumitomo Pharma, Eisai, Eli Lilly, Fuji Film RI Pharma, Janssen Pharmaceutical, Mochida Pharmaceutical, MSD, Nippon Chemipher, Novartis Pharma, Ono Yakuhin, Otsuka Pharmaceutical, Pfizer, Takeda Yakuhin, Tsumura and Yoshitomi Yakuhin within the past 3 years. A. Graff-Guerrero has received research support from the following external funding agencies: CIHR, the US National Institutes of Health, the Ontario Mental Health Foundation, the National Alliance for Research on Schizophrenia and Depression, Mexico Instituto de Ciencia y Tecnología del Distrito Federal, Consejo Nacional de Ciencia y Tecnología, the Ministry of Economic Development and Innovation of Ontario, the Ontario Academic Health Science Center Alternate Funding Plan Innovation Fund and the W. Garfield Weston Foundation. M. Chakravarty has received research funding from the Weston Brain Institute, the Alzheimer's Association and the Michael J. Fox Foundation. He is currently receiving support from the CIHR, the National Sciences and Engineering Research Council of Canada and McGill University's Healthy Brains for Healthy Lives Initiative. He is an associated editor of JPN, but was not involved in the review or decision to accept this paper for publication. S. Nakajima has received grants from the Japan Society for the Promotion of Science, AMED, the Japan Research Foundation for Clinical Pharmacology, the Naito Foundation, the Takeda Science Foundation, the Uehara Memorial Foundation and the Daiichi Sankyo Scholarship Donation Program within the past 3 years, as well as research support, manuscript fees or speaker's honoraria from Dainippon Sumitomo Pharma, Meiji-Seika Pharma, Otsuka Pharmaceutical, Shionogi and Yoshitomi Yakuhin within the past 3 years. No other competing interests declared.

Contributors: All authors designed the study. E. Plitman, R. Tarumi, Y. Iwata, S. Tsugawa, J. Kim, S. Honda, A. Graff-Guerrero and S. Nakajima acquired the data, which all authors analyzed. All authors wrote and reviewed the article. All authors approved the final version to be published and can certify that no other individuals not listed as authors have made substantial contributions to the paper.

Content licence: This is an Open Access article distributed in accordance with the terms of the Creative Commons Attribution (CC BY-NC-ND 4.0) licence, which permits use, distribution and reproduction in any medium, provided that the original publication is properly cited, the use is noncommercial (i.e., research or educational use), and no modifications or adaptations are made. See: https://creativecommons.org/licenses/by-nc-nd/4.0/ 


\section{References}

1. Conley RR, Buchanan RW. Evaluation of treatment-resistant schizophrenia. Schizophr Bull 1997;23:663-74.

2. Howes OD, McCutcheon R, Agid O, et al. Treatment-resistant schizophrenia: Treatment Response and Resistance in Psychosis (TRRIP) working group consensus guidelines on diagnosis and terminology. Am J Psychiatry 2017;174:216-29.

3. Kennedy JL, Altar CA, Taylor DL, et al. The social and economic burden of treatment-resistant schizophrenia: a systematic literature review. Int Clin Psychopharmacol 2014;29:63-76.

4. Seeman P, Kapur S. Schizophrenia: more dopamine, more D2 receptors. Proc Natl Acad Sci U S A 2000;97:7673-5.

5. Iwata $Y$, Nakajima S, Plitman E, et al. Glutamatergic neurometabolite levels in patients with ultra-treatment-resistant schizophrenia: a cross-sectional 3T proton magnetic resonance spectroscopy study. Biol Psychiatry 2019;85:596-605.

6. Tarumi R, Tsugawa S, Noda Y, et al. Levels of glutamatergic neurometabolites in patients with severe treatment-resistant schizophrenia: a proton magnetic resonance spectroscopy study. Neuropsychopharmacology 2020;45:632-40.

7. Fornito A, Yücel M, Dean B, et al. Anatomical abnormalities of the anterior cingulate cortex in schizophrenia: bridging the gap between neuroimaging and neuropathology. Schizophr Bull 2009;35:973-93.

8. Brugger SP, Howes OD. Heterogeneity and homogeneity of regional brain structure in schizophrenia: a meta-analysis. JAMA Psychiatry 2017;74:1104-11.

9. Bush G, Luu P, Posner MI. Cognitive and emotional influences in anterior cingulate cortex. Trends Cogn Sci 2000;4:215-22.

10. Vogt BA, Finch DM, Olson CR. Functional heterogeneity in cingulate cortex: the anterior executive and posterior evaluative regions. Cereb Cortex 1992;2:435-43.

11. Beckmann M, Johansen-Berg H, Rushworth MFS. Connectivitybased parcellation of human cingulate cortex and its relation to functional specialization. J Neurosci 2009;29:1175-90.

12. Palomero-Gallagher N, Hoffstaedter F, Mohlberg H, et al. Human pregenual anterior cingulate cortex: structural, functional, and connectional heterogeneity. Cereb Cortex 2019;29:2552-74.

13. van Heukelum S, Mars RB, Guthrie M, et al. Where is cingulate cortex? A cross-species view. Trends Neurosci 2020;43:285-99.

14. Mouchlianitis E, Bloomfield MAP, Law V, et al. Treatment-resistant schizophrenia patients show elevated anterior cingulate cortex glutamate compared to treatment-responsive. Schizophr Bull 2016;42:744-52.

15. Egerton A, Murphy A, Donocik J, et al. Dopamine and glutamate in antipsychotic-responsive compared with antipsychotic-nonresponsive psychosis: a multicenter positron emission tomography and magnetic resonance spectroscopy study (STRATA). Schizophr Bull 2021;47:505-16.

16. Demjaha A, Egerton A, Murray RM, et al. Antipsychotic treatment resistance in schizophrenia associated with elevated glutamate levels but normal dopamine function. Biol Psychiatry 2014;75:e11-3.

17. Lahti AC, Reid MA. Is there evidence for neurotoxicity in the prodromal and early stages of schizophrenia? Neuropsychopharmacology 2011;36:1779-80.

18. Mehta A, Prabhakar M, Kumar P, et al. Excitotoxicity: bridge to various triggers in neurodegenerative disorders. Eur J Pharmacol 2013;698:6-18.

19. Kritis AA, Stamoula EG, Paniskaki KA, et al. Researching glutamateinduced cytotoxicity in different cell lines: a comparative/ collective analysis/study. Front Cell Neurosci 2015;9:91.

20. Patel R, Steele CJ, Chen AGX, et al. Investigating microstructural variation in the human hippocampus using non-negative matrix factorization. Neuroimage 2020;207:116348.
21. Sotiras A, Resnick SM, Davatzikos C. Finding imaging patterns of structural covariance via non-negative matrix factorization. Neuroimage 2015;108:1-16.

22. Sotiras A, Toledo JB, Gur RE, et al. Patterns of coordinated cortical remodeling during adolescence and their associations with functional specialization and evolutionary expansion. Proc Natl Acad Sci U S A 2017;114:3527-32.

23. Tardif CL, Gauthier CJ, Steele CJ, et al. Advanced MRI techniques to improve our understanding of experience-induced neuroplasticity. Neuroimage 2016;131:55-72.

24. Ochi R, Noda Y, Tsuchimoto S, et al. White matter microstructural organizations in patients with severe treatment-resistant schizophrenia: a diffusion tensor imaging study. Prog Neuropsychopharmacol Biol Psychiatry 2020;100:109871.

25. Shah P, Plitman E, Iwata Y, et al. Glutamatergic neurometabolites and cortical thickness in treatment-resistant schizophrenia: implications for glutamate-mediated excitotoxicity. J Psychiatr Res 2020;124:151-8.

26. Kim J, Plitman E, Iwata Y, et al. Neuroanatomical profiles of treatment-resistance in patients with schizophrenia spectrum disorders. Prog Neuropsychopharmacol Biol Psychiatry 2020;99:109839.

27. Kay SR, Fiszbein A, Opler LA. The positive and negative syndrome scale (PANSS) for schizophrenia. Schizophr Bull 1987;13: 261-76.

28. Guy W, editor. ECDEU assessment manual for psychopharmacology. Rockville (MD): US Department of Health, Education, and Welfare; 1976.

29. Randolph C, Tierney MC, Mohr E, et al. The Repeatable Battery for the Assessment of Neuropsychological Status (RBANS): preliminary clinical validity. J Clin Exp Neuropsychol 1998;20:310-9.

30. Tustison NJ, Avants BB, Cook PA, et al. N4ITK: improved N3 bias correction. IEEE Trans Med Imaging 2010;29:1310-20.

31. Veraart J, Novikov DS, Christiaens D, et al. Denoising of diffusion MRI using random matrix theory. Neuroimage 2016;142: 394-406.

32. Andersson JLR, Sotiropoulos SN. An integrated approach to correction for off-resonance effects and subject movement in diffusion MR imaging. Neuroimage 2016;125:1063-78.

33. Tournier J-D, Smith R, Raffelt D, et al. MRtrix3: a fast, flexible and open software framework for medical image processing and visualisation. Neuroimage 2019;202:116137.

34. Veraart J, Sijbers J, Sunaert S, et al. Weighted linear least squares estimation of diffusion MRI parameters: strengths, limitations, and pitfalls. Neuroimage 2013;81:335-46.

35. Provencher SW. Automatic quantitation of localized in vivo $1 \mathrm{H}$ spectra with LCModel. NMR Biomed 2001;14:260-4.

36. Wijtenburg SA, Knight-Scott J. Very short echo time improves the precision of glutamate detection at $3 \mathrm{~T}$ in $1 \mathrm{H}$ magnetic resonance spectroscopy. J Magn Reson Imaging 2011;34:645-52.

37. Zhang Y, Shen J. Simultaneous quantification of glutamate and glutamine by J-modulated spectroscopy at 3 Tesla. Magn Reson Med 2016;76:725-32.

38. Klein A, Tourville J. 101 labeled brain images and a consistent human cortical labeling protocol. Front Neurosci 2012;6:171.

39. Yang Z, Oja E. Linear and nonlinear projective nonnegative matrix factorization. IEEE Trans Neural Netw 2010;21:734-49.

40. Inagaki A, Inada T. Dose equivalence of psychotropic drugs: 2006-version. Jpn J Clin Psychopharmacol 2006;9:1443-7.

41. van Erp TGM, Walton E, Hibar DP, et al. Cortical brain abnormalities in 4474 individuals with schizophrenia and 5098 control subjects via the Enhancing Neuro Imaging Genetics through Meta Analysis (ENIGMA) consortium. Biol Psychiatry 2018;84: 644-54. 
42. Shepherd AM, Laurens KR, Matheson SL, et al. Systematic metareview and quality assessment of the structural brain alterations in schizophrenia. Neurosci Biobehav Rev 2012;36:1342-56.

43. Haijma SV, Van Haren N, Cahn W, et al. Brain volumes in schizophrenia: a meta-analysis in over 18000 subjects. Schizophr Bull 2013; 39:1129-38.

44. Sellgren CM, Gracias J, Watmuff B, et al. Increased synapse elimination by microglia in schizophrenia patient-derived models of synaptic pruning. Nat Neurosci 2019;22:374-85.

45. Radhakrishnan R, Skosnik PD, Ranganathan M, et al. In vivo evidence of lower synaptic vesicle density in schizophrenia. Mol Psychiatry 2021 June 16 [Epub ahead of print]. doi: 10.1038/s41380-021-01184-0.

46. Onwordi EC, Halff EF, Whitehurst T, et al. Synaptic density marker SV2A is reduced in schizophrenia patients and unaffected by antipsychotics in rats. Nat Commun 2020;11:246.

47. Limongi R, Jeon P, Mackinley M, et al. Glutamate and dysconnection in the salience network: neurochemical, effective connectivity, and computational evidence in schizophrenia. Biol Psychiatry 2020;88:273-81.

48. Samanaite R, Gillespie A, Sendt K-V, et al. Biological predictors of clozapine response: a systematic review. Front Psychiatry 2018;9:327.
49. Weston PSJ, Simpson IJA, Ryan NS, et al. Diffusion imaging changes in grey matter in Alzheimer's disease: a potential marker of early neurodegeneration. Alzheimers Res Ther 2015;7:47.

50. Tronchin G, Akudjedu TN, Ahmed M, et al. Progressive subcortical volume loss in treatment-resistant schizophrenia patients after commencing clozapine treatment. Neuropsychopharmacology 2020;45:1353-61.

51. Al-Amin MM, Uddin MMN, Reza HM. Effects of antipsychotics on the inflammatory response system of patients with schizophrenia in peripheral blood mononuclear cell cultures. Clin Psychopharmacol Neurosci 2013;11:144-51.

52. Nakajima S, Takeuchi H, Plitman E, et al. Neuroimaging findings in treatment-resistant schizophrenia: a systematic review. Lack of neuroimaging correlates of treatment-resistant schizophrenia. Schizophr Res 2015;164:164-75.

53. Pierpaoli C, Basser PJ. Toward a quantitative assessment of diffusion anisotropy. Magn Reson Med 1996;36:893-906.

54. Nazeri A, Schifani C, Anderson JAE, et al. In vivo imaging of gray matter microstructure in major psychiatric disorders: opportunities for clinical translation. Biol Psychiatry Cogn Neurosci Neuroimaging 2020;5:855-64. 